\title{
A Study on Training Data Selection Method for EEG Emotion Analysis using Machine Learning Algorithm
}

\author{
Jong-Seob Yun ${ }^{1}$ and Jin Heon Kim ${ }^{2 *}$ \\ ${ }^{1}$ Department of Electronics \& Computer Engineering, Seokyeong University \\ ${ }^{2 *}$ Department of Computer Engineering, Seokyeong University \\ ${ }^{1}$ cjsgkwkd4@naver.com,2*jinheon@skuniv.ac.kr
}

\begin{abstract}
Machine learning is a subset of artificial intelligence which tackles problems by creating a learnable model instead of an explicit algorithm for a given issue. Machine learning is widely used in the areas of disease detection, computer vision, and data prediction. Examples of machine learning algorithms include but are not limited to artificial neural networks, $k-N N$, and SVM. The 'learning' of the machine learning model is done via a process of updating the internal parameters using training data set. At this time, the convergence speed of the model and the recognition rate differ depending on the configuration of the learning data. In this paper, we investigate how to select sets of training data in order to improve recognition rate in constructing machine learning models for emotion classification using DEAP (Database for Emotion Analysis using Physiological Signals). EEG was recorded through two channels of FP1 and FP2, selected from among 32 channels during the viewing of images which cause pleasure, sadness, or shock. PSD (Power Spectral Density) values obtained by applying FFT (Fast Fourier Transform) to EEG data were used as the featured data of brain waves for the purpose of being input into an artificial neural network. Finally, emotion classification was performed by modeling the artificial neural network, $k-N N$, and SVM models by selecting training data from EEG data based on Valence and Arousal values determined using the SAM (Self-Assessment Manikin) method.
\end{abstract}

Keywords: Artificial Neural Network, DEAP, EEG, Emotion Analysis, PSD

\section{Introduction}

Electroencephalography (EEG) is a minute current created by signal transmission between the cranial nerves, and is recorded by amplifying the signals observed on the electrodes placed along the scalp. EEG measurements use less expensive equipment and exhibit faster response times than other brain activity detection methods, such as CT scans and MRIs. Recently, because of the advantages of using non-invasive and light equipment, brain waves are being used as a tool to analyze brain activity in a number of fields. EEG is used in the study of human emotion recognition [1], and it is used in the field of diseases such as seizure detection [2], depression diagnosis [3] and BCI. Studies in the field of disease include studies of degenerative brain diseases such as dementia [4], studies of brain dysfunction with motor impairment, and the detection of sleeping disorders [5]. In the BCI field, which allows the user to manipulate the machine by using pre-processed EEG signals, research has been done on robot control using EEG [6-7] and motion control of EIS using EEG [8]. In addition, active research has been conducted on VR content, such as VR and EEG combining to improve user's sense of operation in VR

Received (April 16, 2018), Review Result (June 17, 2018), Accepted (June 20, 2018)

* Corresponding Author 
environment [9], studying language processing [10] and workload recognition in a virtual driving environment [11].

In the emotion classification study using EEG data, there is an issue in that there is an observed difference in the change of the EEG due to the size of the emotion felt for the same emotional stimulus according to the personality or disposition of the subjects. Due to this problem, the independent model for the subject is more difficult to classify due to the volatility of the subjects compared to the subject-dependent model [12].

In this paper, we propose a method for selecting recorded EEG data from subjects with various tendencies when choosing learning data to model the classifier in order to find solutions to these problems. In the proposed method, Valence and Arousal values measured with SAM (Self-Assessment Manikin) were used to classify data for modeling. The EEG data recorded from the subjects who had seen the same stimulus video were divided into two groups using Valence and arousal values, and the learning data was then selected and modeled for each group. The artificial neural network, k-NN, and SVM models, which were widely used for EEG biometric recognition, all were implemented as machine learning models to be learned using training data [13].

The composition of this paper is as follows. Chapter 2 introduces the research related to this paper, and Chapter 3 explains the EEG database used in the experiment. Chapter 4 introduces the training data selection method proposed in this paper. Chapter 5 shows the experimental method and experimental results, and finally is concluded in Chapter 6.

\section{Related Work}

There are many bodies of research pertaining to emotion analysis using frequency transformation of EEG. In [15], EEG data recorded from 15 EEG channels were preprocessed using an FIR filter to remove noise, and the ICA method was used to remove artifacts caused by subjects blinking. Then, an FFT analysis was performed to extract features by frequency spectrum power spectrum analysis. Following that, the extracted feature data were used for inputs into the SVM and K-means classifier for the classification of emotion.

In [16], the DEAP database was used as EEG data, and features were extracted using a bispectral analysis, which detects the phase relationship between frequency components and provides a method of obtaining the phase information through the characterization of any non-Gaussian information included in the EEG signal. Then, experiments were performed to classify emotions using the extracted features as inputs to SVM and artificial neural network classifier.

In [17], the implementation and classification of the intended control device for autonomous navigation by analyzing EEG signals was targeted. EEG data recorded from the 4 frontal F7, AF3, AF4, and F8 channels were pre-processed, normalized, and then feature extraction was performed using the peak picking algorithm. Next, the extracted feature data were used as inputs to the KNN and ANN models, and the data was classified following learning.

In the pre-revision paper [18], EEG data of DEAP, which is a public brain wave database, was frequency-converted using FFT and PSD values that are calculated to extract features. Then, the training data selection method was verified through emotion classification by using extracted feature data as an input value of artificial neural network. In this paper, we extend the experiment of that paper and compare the results with machine learning classifiers other than artificial neural networks.

\section{EEG Database}

In this paper, we use DEAP (Database for Emotion Analysis using Physiological Signals), a brain wave data set prepared for emotion classification research at Queen Mary University of London, England. The DEAP database provides information for the analysis 
of human emotions measured from subjects such as EEG data, galvanic skin response (GSR), electro-oculography (EOG), and body temperature.

EEG data provided by the DEAP database was collected using a sampling frequency of $512 \mathrm{~Hz}$, and then downsampled to a $128 \mathrm{~Hz}$ sampling frequency. The downsampled EEG data is processed through a preprocessing process, such as the application of a bandpass filter that passes a $4-45 \mathrm{~Hz}$ signal, and the removal of signal noise caused by blinking, heart movement, and the like. Biological signals were collected from 32 healthy volunteers aged 19 to 37 years. After showing them 40 one-minute music video clips that stimulate emotions, their emotional state during watching each video was evaluated using SAM (Self-Assessment Manikins), which is used as a self-diagnostic scale [14]. In this paper, we used data recorded on two channels, FP1 and FP2, among the EEG data generated while viewing three emotion-induced images of pleasure, sadness, and shock in the DEAP database.

PSD (Power Spectral Density) values were used to extract feature data for emotion analysis from recorded EEG data. To obtain the PSD value, an algorithm such as Fast Fourier Transform must be used to convert EEG data, which is time domain data, into frequency domain data. Fast Fourier transform is an algorithm designed to quickly perform discrete Fourier transform and its inverse transform converts a time domain function into a frequency domain function. Assuming that the $x_{0}, \ldots, x_{n-1}$ values are complex numbers, the discrete Fourier transform is defined as follows.

$$
\mathrm{X}\left(\mathrm{f}_{\mathrm{k}}\right)=\sum_{\mathrm{n}=0}^{\mathrm{N}-1} \mathrm{x}_{\mathrm{n}} \mathrm{e}^{-\mathrm{j} 2 \pi \mathrm{nk} / \mathrm{N}} \mathrm{k}=0,1, \cdots, \mathrm{N}-1
$$

If the signal function is converted into the frequency domain by Eq. (1), the proportion of the frequency bands of one signal that cannot be confirmed in the time domain can then be confirmed. When the EEG signal is converted into a frequency domain value and the spectrum is generated, the components of the EEG signal are divided into delta (1-4 Hz), theta $(4-8 \mathrm{~Hz})$, alpha $(8-13 \mathrm{~Hz})$, beta (13-30), and gamma $(30-45 \mathrm{~Hz})$ bands. After that, the change of EEG due to emotional change can be analyzed using the change of each EEG frequency band as a result of emotional stimulation.

The PSD value can be obtained by applying Eq. (2) to EEG data converted into frequency domain by applying Eq. (1).

$$
\mathrm{P}\left(\mathrm{f}_{\mathrm{k}}\right)=\mathrm{E}\left[\left|\mathrm{X}\left(\mathrm{f}_{\mathrm{k}}\right)\right|^{2}\right]
$$

The expected value of the PSD index for each band calculated using Eq. (2) can be used as feature data for the purposes of EEG analysis.

The ANN (Artificial Neural Network), SVM (Support Vector Machine), KNN(KNearest Neighbor) models were used as classifiers for emotion classification using EEG feature data. This is done as the input value of models, PSD (Power Spectral Density) value, which is extracted through frequency analysis of EEG using Fast Fourier Transform Algorithm. The models used three labels to classify three emotions: pleasure, sadness, and shock. Datasets were varied in order to compare the performance between the experiments.

\section{Proposed Method}

We propose a method to classify EEG data based on Valence and Arousal values estimated using the SAM to model artificial neural network using the EEG data of subjects with various tendencies. The mean and standard deviation of the Valence and Arousal values of EEG data recorded while subjects watched the same stimulus are used as the classification criteria. The EEG data is divided into two groups: one with a difference from the mean greater than the standard deviation, and one with such a difference that is less than the standard deviation. Figure 1 shows the Valence and 
Arousal values of the EEG data recorded while showing the video clip that elicits an emotion of shock to the subjects.

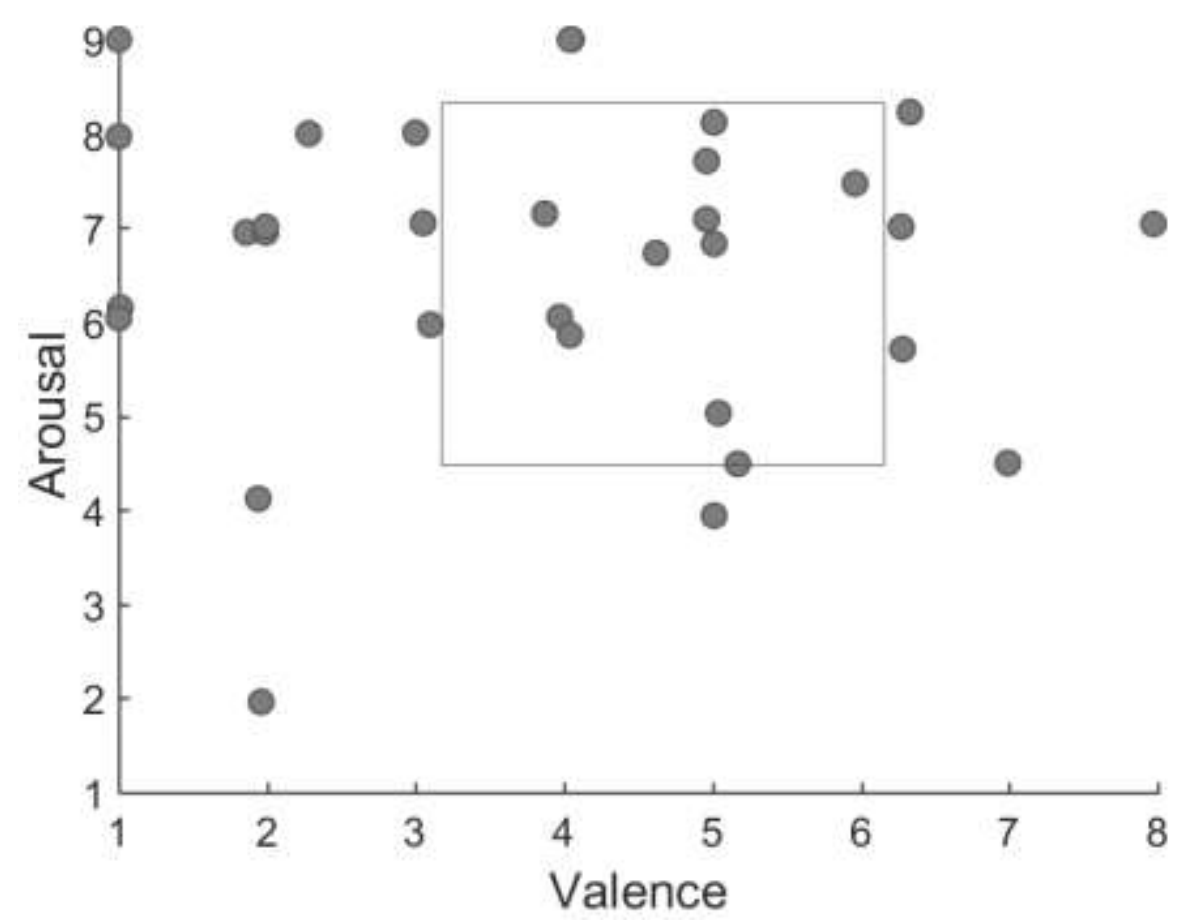

Figure 1. Valence and Arousal Values of Recorded EEG Data while Viewing Shock-Induced Images

In Figure 1, the horizontal axis represents the Valence value of each set of EEG data while the vertical axis represents the Arousal value. The rectangle drawn in the middle of Figure 1. shows the boundary of the EEG data group divided by the mean and standard deviation values of Valence and Arousal. When selecting training data for artificial neural network modeling, the EEG data of subjects with various tendencies can be used for modeling by equally selecting EEG data from each of the two groups.

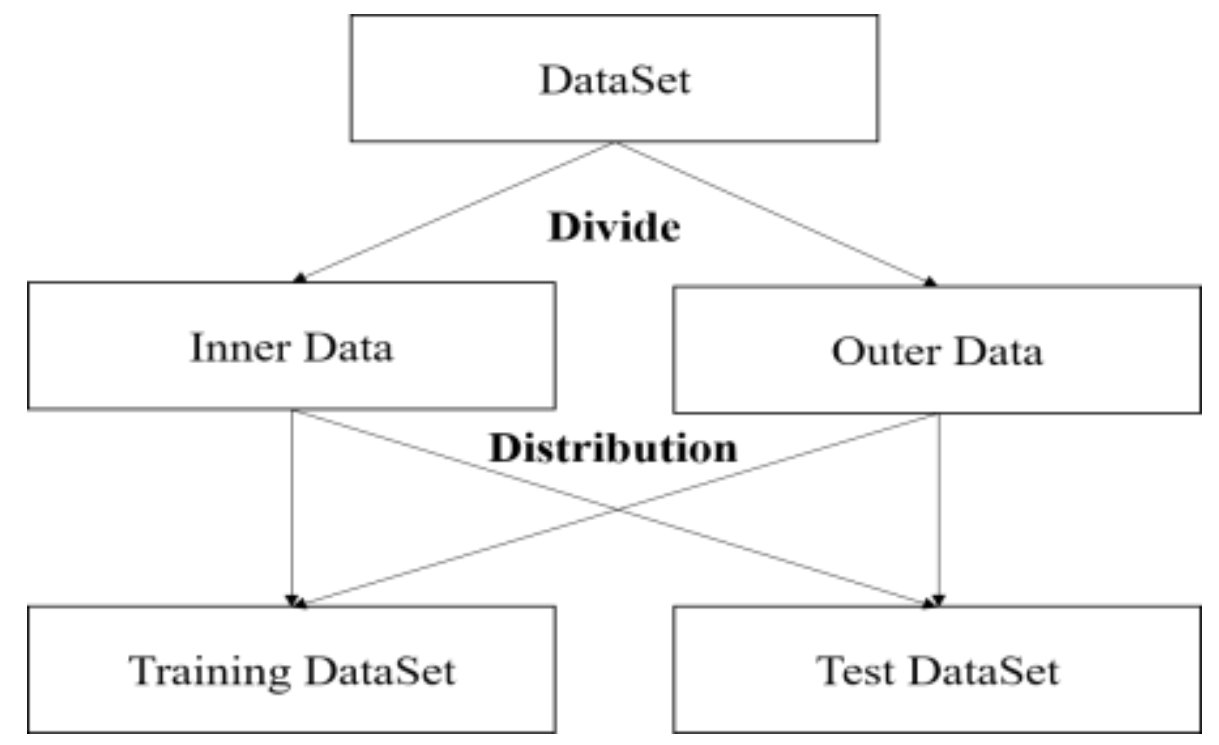

Figure 2. The Proposed Method Flowchart 
Figure 2. displays a flow chart of the proposed method. First, the original data set is divided into separate inner data and outer data groups based on the boundary formed using the average valance, Arousal value, and standard deviation value. Then, the same ratio of data is selected and distributed among the inner and outer data sets to generate both a training data set and a test data set. As a result, both the training data set and the test data set retain the EEG data of subjects with multiple disposition.

In the next section, we conduct experiments to confirm the effects of the proposed training data selection method on the modeling results of the artificial neural network model. Experiments were conducted by dividing experimental data into training data groups and test data groups, which were not used for modeling but only verification.

\section{Experiments and Results}

\subsection{Experiment Dataset}

Figure 3. shows the process of constructing the experimental training data sets in order to confirm the usability of the proposed method. The training data sets are chosen for the purpose of being compared with the proposed method selections of the data in the Valence, Arousal boundary, and the outer sets, respectively. By comparing the results using different data sets as input values of the same classifier, it can be confirmed whether or not the proposed training data selection method shows an advantage in terms of performance improvement.

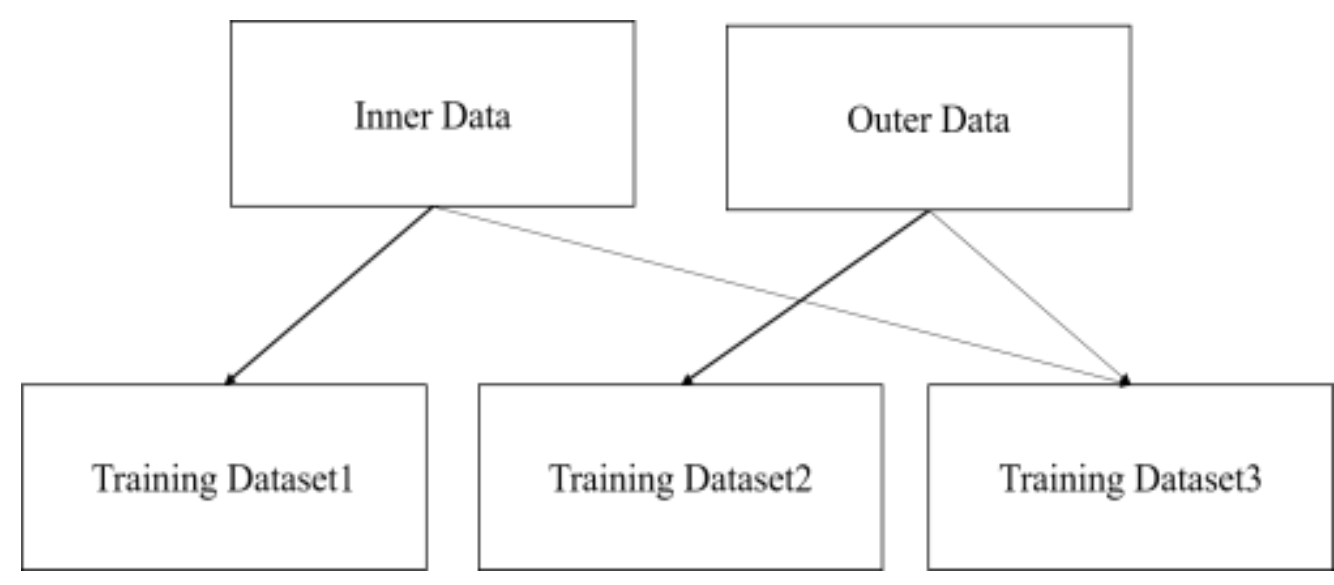

Figure 3. Experimental Dataset Construction Process

\subsection{Classification Algorithms}

\section{Artificial Neural Networks (ANNs)}

The Artificial neural network (ANN) algorithm is a statistical learning algorithm inspired by human brain neurons consisting of connections of multiple neurons. Each neuron in the neural network acts as a classifier that only responds to the input of the previous neuron. Each neuron receives input from a number of other neurons, and derives its output value through an activation function. These results are weighted and converged before the neurons in the neural network arrive at the final decision stage due to their layering. The neural network generally consists of three or more layers, with the addition of one or more hidden layers in addition to the input and output layers. Generally, the data is preprocessed before it is used as an input to the neuron. If large amounts of data are used as neuron inputs without preprocessing, then all the data in the three neuron layers will be uncategorizable. 
In order to process the large amounts of data present in artificial neural networks, we use two or more hidden layers in the neural network so as to increase the connections between the neurons, which is called a deep neural network. In our experiment, we will use only one hidden layer of the neural network structure because we will make use of only a small amount of data.

\section{K-Nearest Neighbors (KNN)}

The KNN algorithm is one of the algorithms commonly used for EEG based pattern classification. The basic principle of the KNN algorithm is to draw comparison of the similarity or distance between the template feature sample and the test sample. The test dataset can be queried for $\mathrm{k}$ samples closest to the feature space and determined via the majority voting method of decision. If $\mathrm{k}$ is set to 1 , the algorithm chooses the closest feature and proceeds with the decision [13]. Classification using the KNN algorithm is sensitive to the local distribution of training samples, and local sensitivity can lead to potential performance instability. Depending on the value of the parameter ' $\mathrm{k}$ ', it can be biased to a dense class or sensitive to noise in the data [13]. However, the KNN algorithm has an explicitly defined learning process, and it is advantageous in that it can be directly compared with the feature samples through storing with the class label without preprocessing the data samples. In this paper, we conduct the experiment with KNN model with parameter $\mathrm{k}$ set to 1 .

\section{Support Vector Machine (SVM)}

SVM (Support Vector Machine) is an algorithm with maximum margin hyperplane, which has better performance than linear and nonlinear algorithms. SVM does not locate a hyperplane that separates only the points, but a hyperplane with the largest margin among the many hyperplanes that potentially separate the points [15]. The first of the reasons why the SVM classifier considers the maximum margin when searching for the classification interface between different classes is that it can be operated most stably with the best ability (reducing the curse of the dimension), and secondly because small errors can be minimized when the maximum margin is set. SVM is a learning method to approach problems via a top-down method. It is faster than other learning methods which employ bottom-up method, and it can process larger amounts of data [15]. Unlike the ANN and KNN algorithms, where a single model can have multiple classification labels, SVM cannot have more than one. Therefore, in this project, the SVM that classifies each of the three emotions was constructed, and the three results were confirmed and classified.

\subsection{Experiment Results}

Accuracy, Sensitivity, Specificity, and Precision are the indicators used for verifying the performance of the learned machine learning models. The following formulas show the definitions of each index.

$$
\begin{aligned}
& \text { Accuracy }=\frac{T P+T N}{T P+T N+F P+F N} \\
& \text { Sensitivity }=\frac{T P}{T P+F N} \\
& \text { Specificity }=\frac{T N}{T N+F P} \\
& \text { Precision }=\frac{T P}{T P+F P}
\end{aligned}
$$

In the above equation, TP, TN, FP, and FN are acronyms for True Positive, True Negative, False Positive, and False Negative, respectively. In this paper, we sought to compare the results using the Accuracy index. 
In order to compare and analyze the performance of models modeled using training data selected with the proposed method, we constructed a total of three training data sets. In the first data set, only EEG data in which the Valence and Arousal values are inside the boundaries of the two groups classified by the proposed method were selected. In the second data set, the same with Valence and Arousal values outside the boundary were selected. Finally, in the third data set, the training data was composed by selecting data equally from the two groups. Then, the frequency conversion using Eq. (1), and the calculation of the PSD value using Eq. (2) were performed on the data of each group. In this experiment, we used two channels of FP1 and FP2, so a total of eight input values were generated.

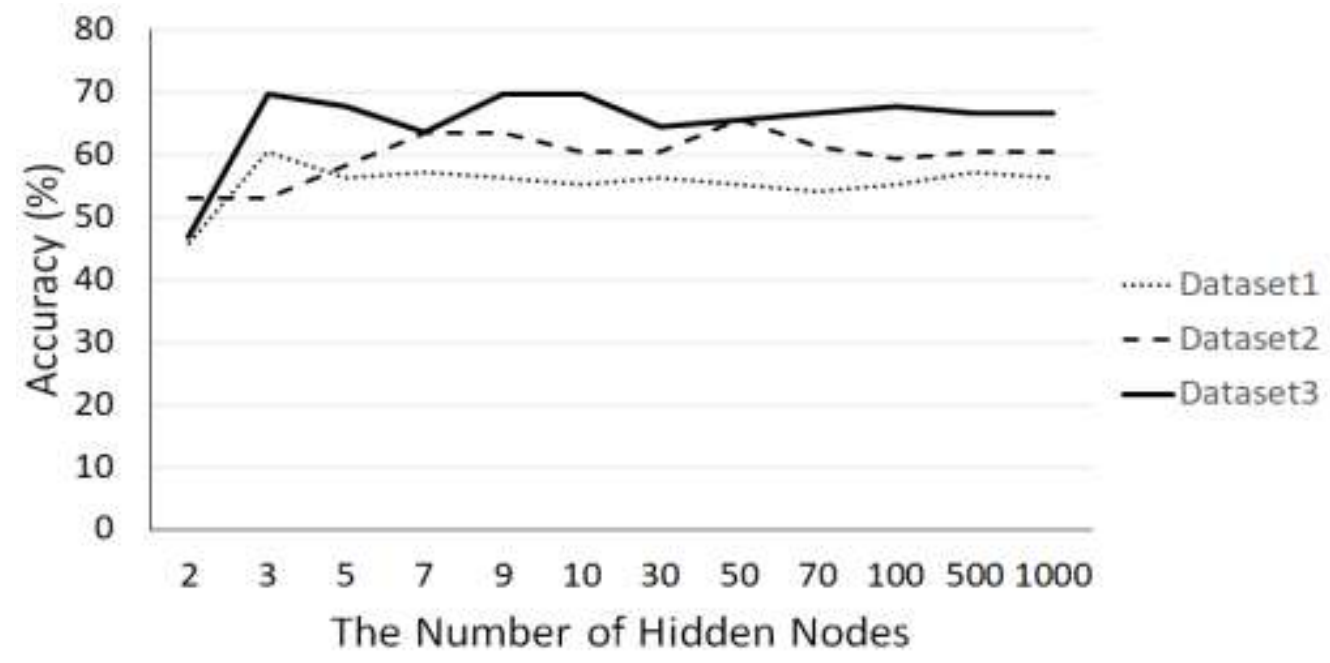

\section{Figure 4. Accuracy based on Learning Data and the Number of Hidden Nodes}

Figure 4 is a graph that shows the changes in accuracy in accordance with the changes in the number of hidden nodes and the dataset in the artificial neural network. In Figure 4, the horizontal axis of the graph represents the number of hidden nodes of each artificial neural network model, and the vertical axis represents the accuracy of each model. The accuracy graph of the neural network model modeled using each dataset is displayed with different lines. Experimental results: When the training data of the neural network model is constructed using the third data set consisting of the EEG data of subjects with various tendencies, it is observed that a higher accuracy is attained than in models using other data sets. As well, it can be confirmed that performance is best when the number of nodes of the artificial neural network is found to be one of 3,9 , or 10 .

Table 1. Accuracy of Classifiers for each Data Set

\begin{tabular}{|c|c|c|c|}
\hline Classifier & Dataset1 & Dataset2 & Dataset3 \\
\hline ANN & 60 & 65 & 70 \\
\hline KNN & 58.33 & 62.50 & 64.74 \\
\hline SVM & 53.12 & 61.46 & 60.52 \\
\hline
\end{tabular}

Table 1 shows the accuracy of the classifiers for each data set. The experimental results show that the accuracy of the ANN and KNN classifiers are the highest when using data selected while using the proposed method. However, when using the SVM classifier, the highest level of accuracy was observed in data set 2. The ANN classifier and the KNN 
classifier with the $\mathrm{k}$ parameter set to 1 produced output among the featured data with the most similar values. However, in the SVM classifier, the result is different from the other two classifiers owing to that it draws the conclusion through the use of data within the range set by the margin, not the nearest one. Due to the differences in the algorithms of these models, there are different influences on the same data set, and it is presumed that the results are correspondingly different.

\section{Conclusion}

In this paper, we classify the EEG data into two groups, near from mean, and far from mean through the use of the Valence and Arousal values of EEG data obtained via the SAM method. Then, by selecting the data equally among the two groups, EEG data recorded from subjects with various tendencies was selected to serve as the training data of the neural network. The mean PSD value of each frequency band of EEG was used as feature criteria to use the selected learning data for the modeling of the artificial neural network model. The EEG data used for the experiment were recorded through the two EEG channels of FP1 and FP2, as well as four bands of theta, alpha, beta, and gamma. Since four features are extracted for each channel, a total of eight values are generated as input values for the three machine learning models.

Following the above, experiments were conducted to effect a comparison of the accuracy of models modeled using the training data selected using the proposed method and the modeled models using EEG data of the subjects with specific tendencies as training data. The experimental results show that the ANN and KNN models with $\mathrm{k}$ parameter set to 1 exhibit the highest accuracy when learned using the training data constructed through the proposed method. For the SVM model, however, data set 2 employing out-of-bounds data as the training data showed the highest accuracy. This is presumed, unlike in the KNN and ANN models, to have relatively similar structures, in turn showing output of the same results among the most similar data used in the SVM model, and it is possible to select various data to draw conclusions according to the margin values.

The experimental results show that the proposed method exhibits positive results when used with ANN and KNN classifiers. However, the results may not be good in an experiment in which EEG data recorded with the same stimulus has no observable significant difference, or subjects with similar tendencies do not participate.

However, if the experimental data group does not fit the above criteria, and the amount of data is small and the results greatly vary according to the training data selected for neural network modeling, then the proposed method can be used as a way to improve model performance.

\section{Acknowledgments}

This research is supported by the Ministry of Culture, Sports and Tourism (MCST), and the Korea Creative Content Agency (KOCCA) via the Culture Technology (CT) Research \& Development Program 2017.

This paper is a revised and expanded version of a paper entitled [A Study on Training Data Selection Method for EEG Emotion Analysis Using Artificial Neural Network] presented at [The 15th 2018 International Interdisciplinary Workshop Series, Hanbat National University. Daejeon. Korea., April 5-7, 2018].

\section{References}

[1] W. Zheng, "Multichannel EEG-Based Emotion Recognition via Group Sparse Canonical Correlation Analysis", IEEE Transactions on Cognitive and Developmental Systems, vol. 9, no. 3, (2017), pp. 281290. 
[2] M. Z. Ahmad, M. Saeed, S. Saleem and A. M. Kamboh, "Seizure detection using EEG: A survey of different techniques", Proceedings of the 2016 International Conference on Emerging Technologies (ICET), Islamabad, Pakistan, (2016), pp. 1-6.

[3] M. Bachmann, J. Lass and H. Hinrikus, "Single channel EEG analysis for detection of depression", Biomedical Signal Processing and Control, vol. 31, (2017), pp. 391-397.

[4] Y. Watanabe, Y. Kobayashi, M. Tanaka, T. Asada, K. Ishii and T. Yagi, "Analysis for Alzheimer's disease using cross-correlation of EEG data", Proceeding of the 10th Biomedical Engineering International Conference (BMEiCON), Hokkaido, Japan, (2017), pp. 1-5.

[5] S. Bisgaard, B. Duun-Christensen, L. Kempfner, H. B. D. Sorensen and P. Jennum, "EEG recordings as a source for the detection of IRBD", Proceeding of the 37th Annual International Conference of the IEEE Engineering in Medicine and Biology Society (EMBC), Milan, Italy, (2015), pp. 606-609.

[6] D. E. Kim, T. J. Lee, S. M. Park, K. E. Ko and K. B. Sim, "EEG Analysis Following Change in Hand Grip Force Level for BCI Based Robot Arm Force Control", Journal of Korean Institute of Intelligent Systems, vol. 23, no. 2, (2013), pp. 172-177.

[7] Y. H. Kim, K. E. Ko, S. M. Park and K. B. Sim, "Practical Use Technology for Robot Control in BCI Environment based on Motor Imagery-P300", Journal of Institute of Control. Robotics and Systems., vol. 19, no. 3, (2013), pp. 227-232.

[8] D. E. Kim, J. H. Yu and K. B. Sim, "EEG Feature Classification for Precise Motion Control of Artificial Hand", Journal of Korean Institute of Intelligent Systems, vol. 25, no. 1, (2015), pp. 29-34.

[9] C. Jeunet, L. Albert, F. Argelaguet and A. Lécuyer, ""Do You Feel in Control?": Towards Novel Approaches to Characterise, Manipulate and Measure the Sense of Agency in Virtual Environments", IEEE Transactions on Visualization and Computer Graphics, vol. 24, no. 4, (2018), pp. 1486-1495.

[10] J. Tromp, D. Peeters, A. S. Meyer and P. Hagoort, "The combined use of virtual reality and EEG to study language processing in naturalistic environments", Behavior Research Methods, vol. 50, no. 2, (2018), pp. 862-869.

[11] J. Fan, J. W. Wade, A. P. Key, Z. E. Warren and N. Sarkar, "EEG-Based Affect and Workload Recognition in a Virtual Driving Environment for ASD Intervention", IEEE Transactions on Biomedical Engineering, vol. 65, no. 1, (2018), pp. 43-51.

[12] N. Jatupaiboon, S. P. Ngum and P. Israsena, "Real-Time EEG-Based Happiness Detection System", The Scientific World Journal, vol. 2013, Article ID 618649, (2013), pp. 1-12.

[13] S. Yang and F. Deravi, "On the Usability of Electroencephalographic Signals for Biometric Recognition: A Survey”, IEEE Transactions on Human-Machine Systems, vol. 47, no. 6, (2017), pp. 958-969.

[14] S. Koelstra, C. Muhl, M. Soleymani, J. S. Lee, A. Yazdani, T. Ebrahimi, T. Pun, A. Nijholt and I. Patras, "DEAP: A Database for Emotion Analysis; Using Physiological Signals", IEEE Transactions on Affective Computing, vol. 3, no. 1, (2012), pp. 18-31.

[15] H. J. Lee, D. I. Shin and D. K. Shin, "The Classification Algorithm of Users' Emotion Using BrainWave", The Journal of Korean Institute of Communications and Information Sciences, vol. 39, no. 2, (2014), pp. 122-129.

[16] N. Kumar, K. Khaund and S. M. Hazarika, "Bispectral Analysis of EEG for Emotion Recognition", Procedia Computer Science, vol. 84, (2016), pp. 31-35.

[17] S. S. Poorna, P. M. V. D. Sai Baba, G. L. Ramya, P. Poreddy, L. S. Aashritha, G. J. Nair and S. Renjith, "Classification of EEG based control using ANN and KNN - A comparison", Proceedings of IEEE International Conference on Computational Intelligence and Computing Research (ICCIC), Chennai, India, (2016), pp. 1-6.

[18] J. S. Yun and J. H. Kim, "A Study on Training Data Selection Method for EEG Emotion Analysis Using Artificial Neural Network", International Journal of Hybrid Information Technology, vol. 11, no. 1, (2018), pp. 7-12.

\section{Authors}

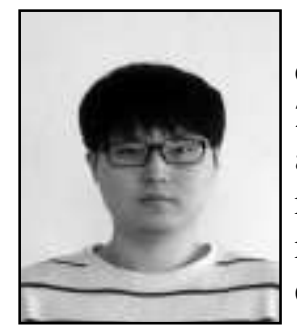

Jong-Seob Yun, Jong-Seob Yun received his B.S. degree in computer engineering from Seokyeong University in Seoul, Korea, in 2017. He is currently working towards an M.S. degree in electronics and computer engineering from the same university. His current research interests include bio signal analysis such as emotion recognition using EEG, and image signal processing such as image enhancement, and their hardware implementations. 


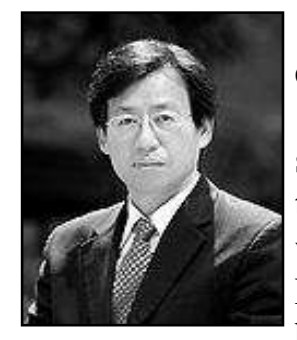

Jin Heon Kim, received his B.S. and M.S. degrees in electrical engineering from Korea University in Seoul, Korea, in 1982 and 1984 respectively, and his Ph.D. in electrical engineering from the same university in 1994. From 1984 to 1986, he was employed with the Oriental Precision Company, in Korea. From 1986 to 1989, he was with the Samsung Advanced Institute of Technology, in Korea. From 1989 to 1990, he was an FAE of Zymos USA at their Korean branch. Since 1995, he has been with the Department of Computer Engineering of Seokyeong University in Seoul, Korea. His current research interests include bio signal analysis such as emotion recognition using EEG, HDR image combining, image enhancement, and their hardware implementations. 